\title{
Gender-related differences in HDL structure with the progression of microalbuminuria in patients with type 2 diabetes
}

\begin{abstract}
Background: The effect of gender on HDL molecules in patients with type 2 diabetes and albuminuria has not been studied sufficiently. We questioned whether HDL-C, Apo A-I and their ratio in the early stages of diabetic nephropathy differs between men and women.

Methods: We designed a matched case-control study of 38 microalbuminuric patients with type 2 diabetes (cases) and 38 age and body mass index matched normoalbuminuric patients with type 2 diabetes (controls). In this study, we investigated HDL-C/Apo A-I ratio as an index of HDL structure and function in clinic. We used diabetic nephropathy as a known model of dyslipidemia and investigated HDL-C, Apo A-I and HDL-C/Apo A-I levels in diabetic patients with microalbuminuria. Because of gender difference in HDL-C levels and cardiovascular risk, gender was considered as an interactive factor.

Results: Apo A-I/HDL-C ratio in female microalbuminuric cases was higher than in the normoalbuminuric controls ( $\mathrm{P}$-value $<0.001$ ), while there was no significant difference in HDL-C or Apo A-I between the two groups. No significant difference in; HDL-C, Apo A-I or Apo A-I/HDL-C ratio, was observed between microalbuminuric and normoalbuminuric males. In a conditional logistic regression model the Apo A-I/ HDL-C ratio was significantly $(\mathrm{P}-\mathrm{value}=0.04$ ) associated with microalbuminuria. Apo A-I/HDL-C ratio and sex interaction also showed a borderline association $(\mathrm{P}-$ Value $=0.05)$.

Conclusion: In patients with type 2 diabetes, measurement of HDL-C and Apo A-I has not conclusively represented the HDL role in albuminuria progression. This is partly due to structural or functional impairment of the HDL particles without any effect on their serum levels, and partly because of different alterations found in males and females. We suggest that such impairments happen faster and more progressively in females.
\end{abstract}

Keywords: type 2 diabetes, nephropathy, HDL molecule, male and female
Volume 2 Issue 3 - 2015

\author{
Manouchehr Nakhjavani,' Mehrdad Larry,' \\ Arash Aghajani Nargesi,' Ebrahim Mostafavi,'2 \\ Hossein Mirmiranpour,' Alireza Esteghamati' \\ 'Tehran University of Medical Sciences, Iran \\ ${ }^{2}$ Department of Laboratory Medicine, Tehran University of \\ Medical Sciences, Iran
}

\begin{abstract}
Correspondence: Manouchehr Nakhjavani, Professor of Endocrinology, Endocrinology and Metabolism Research Center (EMRC), Vali-Asr Hospital,Tehran University of Medical Sciences, Tehran, Iran, P O Box 13 |45-784, Tel (+982 I)-884 I 79I, Fax (+982I)-64432466, Email nakhjavanim@tums.ac.ir
\end{abstract}

Received: June 17, 2015 | Published: July 22, 2015
Abbreviations: HDL, high density lipoprotein; HDL-C, high density lipoprotein cholesterol; Apo A-I, apolipoprotein A-I; ADA, american diabetes association; AST, aspartate transaminase; ALT, alanine transaminase; OCP, oral contraceptive pills; BMI, body mass index; IHD, ischemic heart disease; FBS, fasting blood sugar; HbAlc, hemoglobin a1c; LDL-C, low-density lipoprotein cholesterol; ESR, erythrocyte sedimentation rate; $\mathrm{CV}$, coefficient of variance; IFCC, international federation of clinical chemistry and laboratory medicine; GFR, glomerular filtration rate; MDRD, modification of diet in renal disease; SPSS, statistical package for social science program; ABCA1, atp-binding cassette transporter A1; ABCG1, atp-binding cassette transporter G1; AGE, advanced glycation end products; LCAT, lecithin cholesterol acyl transferase; ApoA-II, apolipoprotein A-II; SR-BI, scavenger receptor class b member 1

\section{Introduction}

HDL-C is an antiatherogenic, anti-inflammatory and antithrombotic particle. ${ }^{1}$ Low levels of HDL-C are associated with chronic lowgrade inflammation and oxidative stress in type 2 diabetic patients with dyslipidemia. ${ }^{2,3}$ This systemic inflammation may change HDL properties into being dysfunctional and proinflammatory. ${ }^{4,5}$

Cholesterol efflux capacity from macrophages, is known to be a metric of HDL antiatherogenic function, and this is independent of HDL cholesterol levels. ${ }^{6}$ It has been suggested that measuring HDL cholesterol levels may not accurately predict the composition and anti-inflammatory properties of HDL. ${ }^{4}$

Apo A-I is a major HDL protein which acquires phospholipids and free cholesterol from peripheral tissues in order to form spherical HDL particles. ${ }^{7-9}$ In vitro studies have shown abnormal compositional changes in HDL and Apo A-I molecules in patients with type 2 diabetes, which impairs its functional properties..$^{9,10}$

In patients with type 2 diabetes, a reduction of HDL-C level is associated with an increased risk of renal injury; ${ }^{11}$ and lower HDL-C can be considered to be a risk factor for developing albuminuria in type 2 diabetes. ${ }^{12}$ The impairment of HDL function has also been investigated in diabetic nephropathy. ${ }^{7,13}$

There are elevated levels of atherogenic lipid particles in diabetic women compared with men. ${ }^{14-16}$ This is an important risk factor for 
cardiovascular events in women. ${ }^{14,17,18}$ In addition, despite the presence of a higher atherogenic lipid profile in women with type 2 diabetes compared with men, ${ }^{14-16}$ its importance in diabetic nephropathy has not been well studied.

Direct measurement of HDL-C or Apo A-I levels in normoalbuminuric and microalbuminuric patients with type 2 diabetes has not been conclusive to show the impairment in HDL particles. ${ }^{12,19-22}$ The purpose of this study was to determine the effect of gender on HDL and Apo A-I levels and the Apo A-I/HDL-C ratio in type 2 diabetes mellitus patients with established microalbuminuria, compared with normoalbuminuric patients.

\section{Materials and methods}

\section{Study patients}

This was a matched case-control study, conducted between February 2014 and December 2014. The study population consisted of 76 participants, including 38 micro aluminuric patients with type 2 diabetes and 38 normoalbuminuric control subjects with type 2 diabetes. Cases and controls were matched for sex (22 males and 16 females in the case group and 22 males and 16 females in the control group) and closely matched for age and BMI. The diabetic patients were recruited from the diabetes clinic of Vali-Asr hospital which is affiliated with Tehran University of Medical Sciences.

In selecting the case group, established microalbuminuria during several 24hour urine collections was taken into consideration. Exclusion criteria for type 2 diabetes patients were; dialysis, glomerulonephritis, AST $>30 \mathrm{U} / \mathrm{L}$, and/or ALT $>40 \mathrm{U} / \mathrm{L}$, hematologic disease, congestive heart failure, stroke or myocardial infarction, autoimmune disease, hormone replacement therapy, OCP use, pregnancy, hospital admission in the previous six months, onset age of diabetes of less than 30 or more than 70years, diabetic ketoacidosis and non-ketotic hyperosmolar state.

All participants gave written informed consent before participation in the study. This study complied with the principles of the declaration of Helsinki. The local ethics review committee of Tehran University of Medical Sciences approved the study protocol.

\section{Clinical characterization}

Diabetes was diagnosed according to ADA criteria. ${ }^{23}$ The ADA criteria were also used for cutoff values of 24 hour urine collection to diagnose microalbuminuria as $30-299 \mathrm{mg} / 24 \mathrm{~h}$ albumin excretion and normoalbuminuria as $<30 \mathrm{mg} / 24 \mathrm{~h}$ albumin excretion..$^{24}$ Demographic and anthropometric data including; age, sex, height and weight were recorded, as well as duration of the diabetes. BMI; $\left(\mathrm{Kg} / \mathrm{m}^{2}\right)$ was calculated according to the Quetelet formula. Blood pressure was measured in a sitting position and remeasured twice after a period of five minutes on average. A thorough investigation of diabetic complications was carried out in patients with diabetes. IHD was defined as; previously known coronary artery disease, positive exercise stress test, or at-rest electrocardiographic findings suggestive for IHD. A complete drug history of the patients was taken for; hypoglycemic drugs, insulin therapy, antihypertensive and lipidlowering drugs. Hypoglycemic drugs were; glyburide and metformin. Antihypertensive drugs were; captopril, enalapril, losartan, atenolol, diltiazem and amiloride. Lipid-lowering drugs were; statins and gemfibrozil.

\section{Biochemical analysis}

Fasting blood samples were taken after 12 hour overnight fasting.
Fresh blood was used for the measurement of; FBS, HbAlc, triglycerides, total cholesterol, LDL-C, creatinine, urea, uric acid, ALT, AST and ESR. Glucose measurements were carried out using the glucose oxidase method (intra-assay CV 2.1\%, inter-assay CV 2.6\%). $\mathrm{HbA} 1 \mathrm{c}$ was determined by high-pressure liquid chromatography.

Total cholesterol, LDL-C and triglycerides levels were determined using direct enzymatic methods (Parsazmoon, Karaj, Iran). Samples were stored in the freezer at a temperature of $-20^{\circ} \mathrm{C}$ for 14 days before HDL-C and Apo A-I measurement. HDL-C was also determined using a direct enzymatic method (Pishtazteb, Tehran, Iran). Apo A1 was measured (Cobas INTEGRA Tina-quant Apo A-1 ver. 2) following the principles of antigen-antibody reaction using the immuno Turbid metric method. (Intra-assay $\mathrm{CV}=0.8 \%$, inter-assay $\mathrm{CV}=1.7 \%$ ) Creatinine was measured using the calibrated Jaffe method (Parsazmoon, Karaj, Iran, intra-assay $\mathrm{CV}=3.3 \%$ ). Urea was measured using a colorimetric assay (Parsazmoon, Karaj, Iran). Analyses of serum ALT and AST were performed using enzymatic photometry by the IFCC method (ALT intra-assay CV $=3.7 \%$, AST intra-assay $\mathrm{CV}=2.5 \%$ ). Uric acid was measured by the calorimetric method (intra-assay $\mathrm{CV}=1.27 \%$ ). ESR was determined using the Westergren method. Patients were instructed in timed 24hour urine collection for measurement of urinary protein excretion. Urine protein was measured by immunoturbidimetry (intra-assay $\mathrm{CV}=2.1 \%$ ), and GFR was calculated using the MDRD formula. ${ }^{23}$

\section{Statistical analysis}

Continuous variables were presented as mean \pm standard error of mean (SEM). Categorical variables are presented as number and percentage. Chi square test and paired sample t-test were used for between group comparisons as indicated. A conditional logistic regression model was employed to evaluate outcomes (microalbuminuria, normoalbuminuria) in the studied population. Input variables were; HDL-C, Apo A-I, Apo A-I/ HDL-C, duration of diabetes, systolic blood pressure, diastolic blood pressure, FBS, $\mathrm{HbA1c}, \mathrm{GFR}$, triglycerides, total cholesterol and LDL-C. P value $<0.05$ was considered as statistically significant. SPSS for windows (version 19; Chicago, IL) was used for the analysis.

\section{Results}

Primary characteristics of the study population are presented in Table 1 . The only observed differences were for plasma uric acid and the number of patients using oral antihypertensive drugs. No significant differences were observed for matching variables (age, sex and BMI) between the two groups (Table 1).

Serum levels of HDL-C, Apo A-1 and Apo A-1/HDL-C in the study groups are presented in Table 2. Basically, there was no significant difference found between the cases and controls in; HDL-C, Apo A-1 or Apo A-1/HDL-C. When comparing within the gender subgroups, there was no significant difference found in; HDL-C, Apo A-1 or Apo A-1/HDL-C between microalbuminuric and normoalbuminuric males. In the female subgroup, patients with microalbuminuria had a higher Apo A-1/HDL-C ratio than the normoalbuminuric controls, while there was no significant difference in HDL-C or Apo A-1 between the two groups (Table 2).

Table 3 shows the results of a conditional logistic regression model considering albuminuria as the outcome variable. In the final model Apo A-1/HDL-C ratio was significantly $(\mathrm{P}=0.036)$ associated with microalbuminuria. The Apo A-1/HDL-C ratio and sex interaction also, showed a borderline association $(\mathrm{P}=0.054)$. Other included variables, however, did not have any significant association with 
microalbuminuria. Figure1 illustrates the interaction between Apo A-1/HDL-C ratio and sex. Figurel suggests that microalbuminuric females have higher Apo A-1/HDL-C ratio compared to their normoalbuminuric counterparts while such association is not observed in the male group.

Table I Baseline Characteristics of study population

\begin{tabular}{|c|c|c|c|}
\hline & Case $(n=38)$ & Control( $(n=38)$ & p-value \\
\hline Sex (male, female) & $(22,16)$ & $(22,16)$ & NS \\
\hline Age (years) & $58.47 \pm 1.69$ & $57.78 \pm|.5|$ & NS \\
\hline $\mathrm{BMI}(\mathrm{kg} / \mathrm{m} 2)$ & $27.15 \pm .74$ & $26.31 \pm .73$ & $<0.0001$ \\
\hline Urine Micro albumin (mg/24h) & $192.44 \pm 20.97$ & $7.49 \pm 1.12$ & NS \\
\hline Duration of diabetes (years) & $10.69 \pm 1.53$ & $9.19 \pm 1.13$ & NS \\
\hline $\mathrm{SBP}(\mathrm{mmHg})$ & $122.42 \pm 3.02$ & $123.13 \pm 3.85$ & NS \\
\hline $\mathrm{DBP}(\mathrm{mmHg})$ & $71.54 \pm 1.63$ & $72.84 \pm 1.79$ & NS \\
\hline FBS (mg/dl) & $207.4 \pm 14.03$ & $196.58 \pm 15.08$ & NS \\
\hline $\mathrm{HbAlc}(\%)$ & $9.10 \pm 0.45$ & $8.62 \pm 0.39$ & NS \\
\hline $\mathrm{HbAlc}(\mathrm{mmol} / \mathrm{mol})$ & $75.92 \pm 4.94$ & $70.67 \pm 4.24$ & NS \\
\hline Creatinine (mg/dl) & $1.15 \pm 0.05$ & $1.07 \pm 0.09$ & NS \\
\hline Urea $(g / 24 h)$ & $35.59 \pm 2.85$ & $33.36 \pm 2.99$ & $<0.05$ \\
\hline Uric Acid (mg/dl) & $5.45 \pm .40$ & $3.80 \pm .45$ & NS \\
\hline Total Cholesterol (mg/dl) & $187.07 \pm 8.53$ & $184.13 \pm 8.85$ & NS \\
\hline LDL-C (mg/dl) & $107.36 \pm 7.04$ & $105.74 \pm 6.79$ & NS \\
\hline Triglycerides (mg/dl) & $190.90 \pm 22.15$ & $163.42 \pm 17.89$ & NS \\
\hline AST (U/L) & $20.96 \pm 1.97$ & $21.10 \pm 1.60$ & NS \\
\hline ALT (U/L) & $26.28 \pm 3.16$ & $23.85 \pm 2.52$ & NS \\
\hline $\mathrm{ALP}(\mathrm{U} / \mathrm{L})$ & $278.50 \pm 99.48$ & $157.33 \pm 27.87$ & NS \\
\hline GFR (ml/min) & $65.6 I \pm 3.04$ & $77.67 \pm 6.37$ & \\
\hline \multicolumn{4}{|l|}{ Medication } \\
\hline Oral Hypoglycemic drug (n (\%)) & $28(74)$ & $33(87)$ & NS \\
\hline Insulin (n (\%)) & $12(31)$ & $8(21)$ & NS \\
\hline Oral Antihypertensive drug (n (\%)) & $23(61)$ & II(29) & $<0.01$ \\
\hline Oral Lipid-lowering drug ((n (\%)) & $25(66)$ & $23(61)$ & NS \\
\hline IHD (n (\%)) & $4(I I)$ & $2(6)$ & NS \\
\hline
\end{tabular}

Data is presented as Mean \pm Standard Error of Mean or frequency and percent. p-values are obtained by paired-samples $t$ test or chi-squared test as appropriate

Table 2 HDL and Apo A-I in study groups according to gender

\begin{tabular}{|c|c|c|c|c|}
\hline & & Case $(n=38)$ & Control $(n=38)$ & p-value \\
\hline \multirow{3}{*}{$\begin{array}{l}\text { HDL-C } \\
(\mathrm{mg} / \mathrm{dl})\end{array}$} & Male & $37.13 \pm 1.86$ & $37.77 \pm 1.83$ & 0.8 \\
\hline & Female & $42.31 \pm 2.26$ & $47.68 \pm 2.93$ & 0.15 \\
\hline & Total & $39.32 \pm 1.48$ & $41.95 \pm 1.80$ & 0.26 \\
\hline \multirow{3}{*}{$\begin{array}{l}\text { Apo A-I } \\
(\mathrm{mg} / \mathrm{dl})\end{array}$} & Male & $|3| .04 \pm 3.7 \mid$ & $138.18 \pm 4.93$ & 0.25 \\
\hline & Female & $|52.3| \pm 8.52$ & $157.56 \pm 8.29$ & 0.66 \\
\hline & Total & $140.00 \pm 4.47$ & $146.34 \pm 4.72$ & 0.33 \\
\hline \multirow{3}{*}{$\begin{array}{l}\text { Apo A-I / } \\
\text { HDL-C }\end{array}$} & Male & $3.6 I \pm 0.10$ & $3.73 \pm 0.12$ & 0.44 \\
\hline & Female & $3.6 I \pm 0.01$ & $3.36 \pm .011$ & $<0.001$ \\
\hline & Total & $3.62 \pm 0.07$ & $3.58 \pm 0.09$ & 0.72 \\
\hline
\end{tabular}

Data is presented as Mean \pm Standard Error of Mean. P-values are obtained by paired-samples $\mathrm{t}$ Test
Table 3 Conditional logistic regression was employed to study variables influencing microalbuminuria in patients with type 2 diabetes and Apo A-I/ HDL-C ratio - sex interaction

\begin{tabular}{lll}
\hline $\mathbf{B}$ & P-value & $\mathbf{9 5 . 0 \%} \mathbf{C I}$ for $\operatorname{Exp}(\mathbf{B})$ \\
\hline 0.24 & NS & $(0.901,1.792)$ \\
-0.874 & NS & $(0.128,1.359)$ \\
-19.236 & 0.036 & $(0.000,0.282)$ \\
0.336 & NS & $(0.911,2.149)$ \\
0.104 & NS & $(0.938,1.313)$ \\
-0.094 & NS & $(0.811,1.022)$ \\
0.079 & NS & $(0.917,1.278)$ \\
0.053 & NS & $(0.949,1.171)$ \\
6.413 & 0.054 & $(0.898,413867.815)$ \\
\hline
\end{tabular}




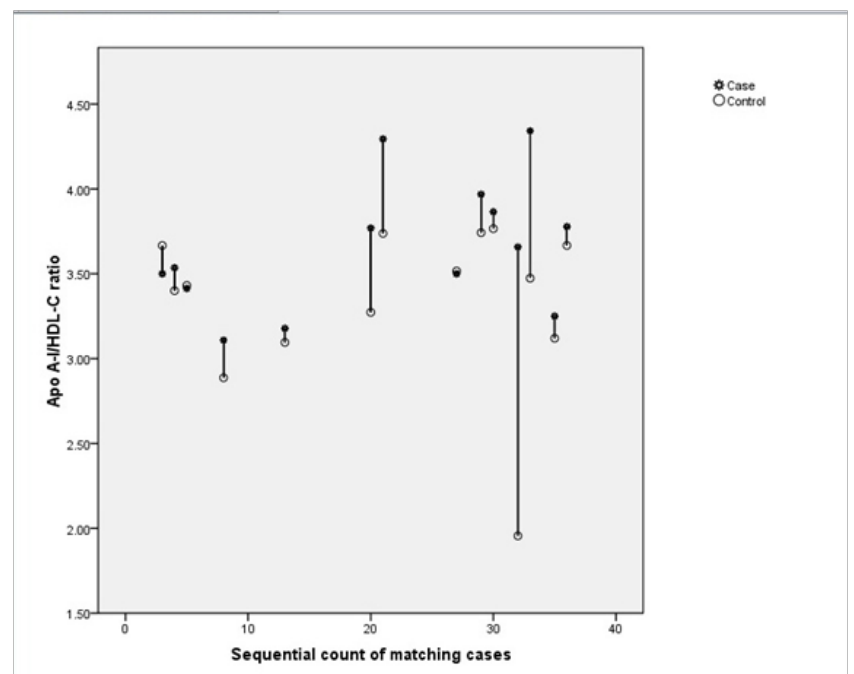

Figure I Comparing Apo A-I/HDL-C ratio in pairs of diabetic females with albuminuria (cases) and without albuminuria (controls), I:I matched by age, sex and BMI.

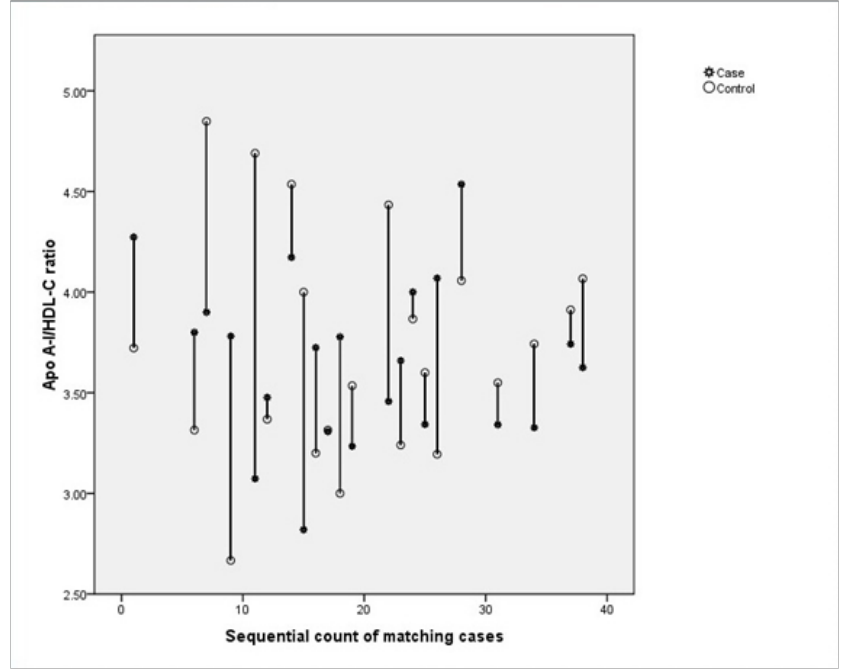

Figure 2 Comparing Apo A-I/HDL-C ratio in pairs of diabetic males with albuminuria (cases) and without albuminuria (controls), I:I matched by age, sex and BMI.

\section{Discussion}

The main finding of the present study was the effect of gender on the Apo A-I/HDL-C ratio-micro albuminuria relationship. The differences between the Apo A-I/ HDL-C ratio in the normoalbuminuric and micro albuminuric groups were significant for females in this study; showing a higher Apo A-I/ HDL-C ratio in the micro albuminuric patients. Such results were not observed in their male counterparts.

In the current study, the Apo A-I/HDL-C ratio was selected as an indicator of HDL structure and somehow its function. This was considered because of conformational changes in HDL molecules in type 2 diabetes. ${ }^{2,9,10,25}$ In a diabetic vascular and oxidative complication such as nephropathy, a lower HDL-C level is a risk factor for microalbuminuria progression, ${ }^{12}$ although the Apo A-I level remains almost intact. ${ }^{22,26}$

However, its anti-inflammatory, antioxidative and antiatherogenic properties are significantly decreased,,$^{2,10,25,27}$ which leads to a higher
Apo A-I/HDL-C ratio. This higher ratio can also predict the presence of smaller HDL particles seen in patients with type 2diabetes, mostly because of their cholesterol and phospholipid depletion and lipid-free Apo A-I. $2,8,28$

This impaired HDL metabolism is partly because of the ABCA1 and ABCG1 altered mechanisms due to the formation of AGEs, and these are associated with diabetic vascular complications. ${ }^{28}$ This leads to a decrease in circulating levels of large, light, cholesterol-rich HDL particles in parallel with a decrease in HDL-C concentrations, whereas levels of small, dense HDL particles are essentially unaltered. ${ }^{2}$ This is similar to patients with coronary disease who generally have smaller, denser HDL particles, leading to the concept that larger HDL particles may be associated with greater protection from coronary heart disease. ${ }^{8}$

In vitro studies show the second important mechanism leading to compositional changes and antioxidant and anti-inflammatory reduction in HDL molecules. Non-enzymatic glycation of Apo A-I in people with type 2 diabetes impairs both the ability of HDL to promote cholesterol efflux from macrophages and its ability to inhibit vascular inflammation. ${ }^{10,25}$

Non-enzymatic glycation of Apo A-I in diabetes mellitus depends on its AGE modification which involves its lysine residues and has considerable responsibility for this impairment. ${ }^{29}$ Since Apo A-I is known as the activator of LCAT, its glycation and thus inactivation cause the production of non-functional LCAT. ${ }^{30}$

Our findings for Apo A-I levels in normoalbuminuric and microalbuminuric patients for both males and females were compatible with previous studies. Such studies compared Apo A-I levels as well as HDL-C in microalbuminuric versus normoalbuminuric patients. Patel et al. in 2012, reported lower HDL-C levels in microalbuminuric patients compared with normoalbuminuric ones, but no such difference was found in Apo A-I levels. ${ }^{21}$ This is the same as the results of Tseng $\mathrm{CH}^{22}$ for 251 normoalbuminuric and 242 microalbuminuric patients. ${ }^{22}$ In 2005 Ridker et al. concluded that apolipoprotein fractions measurement is not an appropriate serum marker for predicting future cardiovascular events. ${ }^{31}$

Winocour et al. ${ }^{32}$ reported a different Apo A-I/ HDL-C ratio in microalbuminuric patients with type 2 diabetes compared with normoalbuminuric ones. ${ }^{32}$ In addition, Soedamah-Muthu et al. ${ }^{33}$ predicted an inverse relationship of HDL-C/ (Apo A-I + Apo A-II) ratio with albuminuria at baseline. ${ }^{33}$ None of these studies discussed a gender effect on the relationship between this ratio and microalbuminuria, but as the current study has shown there is a possibility that functional impairment of HDL is more prominent in females than males in the early stages of diabetic nephropathy.

Women have significantly higher plasma levels of total cholesterol, triglycerides, LDL-C, HDL-C, non-HDL cholesterol, lipoprotein (a) and Apolipoprotein B. ${ }^{15,16}$ This is believed to be an important risk factor for cardiovascular events in females. ${ }^{14,17,18}$ The effect of a worse lipid profile in women on the progression of diabetic nephropathy is less well studied.

The current study showed that a higher Apo A-I/ HDL-C ratio is associated with microalbuminuria in females but not for males. This is consistent with a previous study where we showed that different risk factors influence albuminuria in males and females; thus HDL is considered as a risk factor for women, but not for men. ${ }^{34}$ Furthermore, there is evidence that LCAT activity and LCAT production in women 
with type 2 diabetes is worse compared with men, ${ }^{35}$ which is consistent with the gender effect on HDL functional properties.

The importance of HDL particles in predicting renal damage in type 2 diabetes is still unknown, however, studies in endothelial cell cultures have demonstrated that HDL suppresses the expression of markers of inflammation and cell adhesion molecules in the early stages of diabetic nephropathy, which is compatible with the functional impairment of HDL molecules. ${ }^{11}$ Moreover, Zhou et al. ${ }^{13}$ showed that the capacity of serum to induce ABCA1- and SR-BI -mediated cholesterol efflux is impaired in diabetic patients with incipient or overt nephropathy. ${ }^{13}$

In the current study, no significant relationship was observed between HDL-C levels and microalbuminuria. This is similar to what Shen et al. ${ }^{19}$ reported for HDL-C levels of microalbuminuric and normoalbuminuric groups in a 1069 hospital-based population study; ${ }^{19}$ Perassolo et al. also reported this finding in 2003. ${ }^{20}$ On the contrary, Afghahi et al. ${ }^{12}$ reported a significant difference in HDL-C levels between normoalbuminuric and microalbuminuric patients in 3667 patients with type 2 diabetes; which was lower in the microalbuminuric group. ${ }^{12}$ The diversity of the results in these different reports may be due to the effect of gender on renal damage associated with HDL particles.

\section{Limitations}

Our sample size may be inadequate to predict such exact correlations. As this is a matched case-control study; deciding whether HDL functional properties leads to microalbuminuria or the relationship is inversely accurate remains undetermined. A future cohort study to determine whether such a relationship exists is of the utmost importance.

\section{Conclusion}

In patients with type 2 diabetes, measurements of HDL-C and Apo A-I have not been found to conclusively represent the HDL role in albuminuria progression. This is partly because of functional impairment of Apo A-I as major HDL particle without any effect on its serum level and partly because of different alterations observed in males and females. We suggest such impairment happens faster and more progressively in females.

\section{Acknowledgements}

We are grateful to the staff of Diabetes Research Center and endocrine laboratory of Vali-asr Hospital of Tehran University of Medical Sciences for their assistance.

\section{Authors' contribution}

MN concieved and designed the study, provided expertise and oversight throughout the process, and participated in data collection. ML assisted in data collection, measurements and statistical analyses, conducted the literature review and data interpretations, and drafted the manuscript. AAN performed statistical analyses, assisted in data interpretation and helped to draft the manuscript. EM, HM and $\mathrm{AE}$ participated in design, methodology and coordination of the study. All authors read and approved of the final manuscript.

\section{Conflict of interest}

Author declares that there is no conflict of interest.

\section{References}

1. deGoma EM, deGoma RL, Rader DJ. Beyond High-Density Lipoprotein Cholesterol Levels Evaluating High-Density Lipoprotein Function as Influenced by Novel Therapeutic Approaches. J Am Coll Cardiol. 2008;51(23):2199-2211.

2. Kontush A, Chapman MJ. Why Is HDL Functionally Deficient in Type 2 Diabetes? Curr Diab Rep. 2008;8(1):51-59.

3. Leiter LA, Genest J, Harris SB, et al. Dyslipidemia in Adults With Diabetes. Can J Diab. 2006;30(3):230-240.

4. Navab M, Anantharamaiah GM, Reddy ST, et al. HDL as a Biomarker, Potential Therapeutic Target, and Therapy. Diabetes. 2009;58(12):2711-2717.

5. Ory DS, Schaffer JE. ApoA-1 in Diabetes: Damaged Goods. Diabetes. 2010;59(10):2358-2359.

6. Khera AV, Cuchel M, Llera-Moya Mdl, et al. Cholesterol Efflux Capacity, High-Density Lipoprotein Function, and Atherosclerosis. $N$ Engl J Med. 2011;364(2):127-135.

7. Lapolla A, Brioschi M, Banfi C, et al. Nonenzymatically Glycated Lipoprotein ApoA-I in Plasma of Diabetic and Nephropathic Patients. Ann NY Acad Sci. 2008;1126:295-9.

8. Movva R, Rader DJ. Laboratory Assessment of HDL Heterogeneity and Function. Clin Chem. 2008;54(5):788-800.

9. Gowri MS, Westhuyzen DR Vd, Bridges SR, et al. Decreased Protection by HDL From Poorly Controlled Type 2 Diabetic Subjects Against LDL Oxidation May Be Due to the Abnormal Composition of HDL. Arterioscler Thromb Vasc Biol. 1999;19(9):2226-2233.

10. Nobécourt E, Tabet F, Lambert G, et al. Non-Enzymatic Glycation Impairs the Anti-Inflammatory Properties of Apolipoprotein A-I. Arterioscler Thromb Vasc Biol. 2010;30(4):766-772.

11. Rutledge JC, Ng KF, Aung HH, et al. Role Of Triglyceride-Rich Lipoproteins In Diabetic Nephropathy. Nephrol. 2010;6(6):361-370.

12. Afghahi $\mathrm{H}$, Cederholm J, Eliasson B, et al. Risk factors for the development of albuminuria and renal impairment in type 2 diabetes-the Swedish National Diabetes Register (NDR). Nephrol Dial Transpl. 2010;26(4):1236-1243.

13. Zhou H, Tan KCB, Shiu SWM, et al. Cellular cholesterol efflux to serum is impaired in diabetic nephropathy. Diabetes Metab Res Rev. 2008;24(8):617-623

14. Williams K, Tchernof A, Hunt KJ, et al. Diabetes, Abdominal Adiposity, and Atherogenic Dyslipoproteinemia in Women Compared With Men. Diabetes. 2008;57(12):3289-3296.

15. Schianca GPC P. G Colli E, Bigliocca M, et al. Sex differences in lipid profiles in relation to the progression of glucose abnormalities. $J$ Diabetes. 2012;4(1):95-101.

16. Eriksson M, Zethelius B, Eeg-Olofsson K, et al. Blood lipids in 75048 type 2 diabetic patients: a population-based survey from the Swedish National diabetes register. Eur J Cardiovasc Prev Rehabill. 2010;8:97-105.

17. Mora S, Buring J, Ridker P, et al. Association of HDL Cholesterol With Incident Cardiovascular Events in Women, by LDL Cholesterol and Apo B100 Levels: A Cohort Study. Ann Intern Med. 2011;155(11):742-750.

18. Lee WL, Cheung AM, Cape D, et al. Impact of Diabetes on Coronary Artery Disease in Women and Men: A meta-analysis of prospective studies. Diabetes Care. 2000;23(7):962-968.

19. Shen FC, Chen CY, Su SC, et al. The prevalence and risk factors of diabetic nephropathy in taiwanese type 2 diabetes- a hospital- based study. Acta Nephrol. 2009;23(2):90-95. 
20. Perassolo MS, Almeida JC, Pr'a RL, et al. Fatty Acid Composition of Serum Lipid Fractions in Type 2 Diabetic Patients With Microalbuminuria. Diabetes Care. 2003;26(3):613-618.

21. Patel ML, Sachan R, Gupta KK, et al. Correlation of Plasma Apolipoprotein and lipid profiles with different stages of type 2 diabetic nephropathy- A Hospital based study in North Indian Population. Inter J Scien Res Pub. 2012;2(5):1-6.

22. Tseng CH. Differential dyslipidemia associated with albuminuria in type 2 diabetic patients in Taiwan. Clin Biochem. 2009;42(10-11):1019-1024.

23. American Diabetes Association. Diagnosis and classification of diabetes mellitus. Diabetes Care. 2004;27:S5-S10.

24. American Diabetes Association: Nephropathy in diabetes (Position Statement). Diabetes Care. 2004;27(1):S79-S83.

25. Barter PJ. The Causes and Consequences of Low Levels of High Density Lipoproteins in Patients with Diabetes. Diabetes Metab J. 2011;35(2):101-106.

26. Patel S, Drew BG, Nakhla S, et al. Reconstituted High-Density Lipoprotein Increases Plasma High-Density Lipoprotein AntiInflammatory Properties and Cholesterol Efflux Capacity in Patients With Type 2 Diabetes. J Am Coll Cardiol. 2009;53(11):962-972.

27. Hoang A, Murphy AJ, Coughlan MT, et al. Advanced glycation of apolipoprotein A-I impairs its anti-atherogenic properties. Diabetologia. 2007;50(8):1770-1779.
28. Passarelli M, Tang C, McDonald TO, et al. Advanced Glycation End Product Precursors Impair ABCA1-Dependent Cholesterol Removal From Cells. Diabetes. 2005;54(7):2198-2205.

29. Scanu AM, Edelstein C. HDL: bridging past and present with a look at the future. The FASEB J. 2008;22(12):4044-54.

30. Nobecourt E, Davies MJ, Brown BE, et al. The impact of glycation on apolipoprotein A-I structure and its ability to activate lecithin:cholesterol acyltransferase. Diabetologia. 2007;50(3):643-653.

31. Ridker PM, Rifai N, Cook NR, et al. Non-HDL Cholesterol, Apolipoproteins A-I and B100, Standard Lipid Measures, Lipid Ratios, and CRP as Risk Factors for Cardiovascular Disease in Women. Am Med Assoc. 2005;294(3):326-333.

32. Winocour PH, Harland JOE, Millar JP, et al. Microalbuminuria and associated cardiovascular risk factors in the community. Atherosclerosis. 1992;93(1-2):71-81.

33. Soedamah-Muthu SS, Colhoun HM, Taskinen MR. Differences in HDLcholestrol:apoA-I + apoA-II ratio and apoE phenotype with albuminuric status in type I diabetic patients. Diabetologia. 2000;43(11):1353-1359.

34. Nakhjavani M, Morteza A, Jenab Y, et al. Gender Difference in Albuminuria and Ischemic Heart Disease in Type 2 Diabetes. Clin Med Res. 2012;10(2):51-56.

35. Nakhjavani M, Morteza A, Karimi R, et al. Diabetes induces gender gap on LCAT levels and activity. Life Scien. 2013;92(1):51-54. 